\title{
Acquired hemophilia A presenting as progressive intra-abdominal hemorrhage, muscle hemorrhage and hemothorax postpartum: A case report and literature review
}

\author{
LIYING XU ${ }^{1}$, JUNFA CHEN ${ }^{2}$, XIAJUAN ZHOU $^{3}$, LIJUAN WU ${ }^{1}$, YIXIN TONG ${ }^{1}$, \\ NI ZHU ${ }^{2}$, XIAOMIN HUANG ${ }^{1}$ and ZHUOYI ZHANG ${ }^{1}$ \\ Departments of ${ }^{1}$ Emergency, ${ }^{2}$ Hematology and ${ }^{3}$ General Practice, The First Affiliated Hospital of \\ Zhejiang Chinese Medical University, Hangzhou, Zhejiang 310006, P.R. China
}

Received December 25, 2017; Accepted September 24, 2018

DOI: $10.3892 / \mathrm{etm} .2018 .7031$

\begin{abstract}
Acquired hemophilia A (AHA) is a rare antibody-mediated condition in which autoantibodies form against a coagulation factor, most commonly factor VIII (FVIII), causing severe coagulopathy. Here the present report presents a case of AHA in a 35-year-old postpartum woman with continuous polyserous bloody effusions who was admitted to the First Affiliated Hospital of Zhejiang Chinese Medical University (Hangzhou, China) in October 2017 without a history of trauma, anticoagulation treatment or coagulopathy. At presentation, the patient's hemoglobin level was low (70 g/l; normal range: $115-150 \mathrm{~g} / \mathrm{l}) \mathrm{g} / \mathrm{l}$, blood pressure was $89 / 58 \mathrm{mmHg}$ (normal range, 90-140/60-90 $\mathrm{mmHg}$ ), and activated partial thromboplastin time was $68.4 \mathrm{sec}$ (normal range: $25.0-36.0 \mathrm{sec}$ ), with a normal international normalized ratio (0.94; normal range, $0.8-1.2)$. The reaction time in thrombography was prolonged $(35.8 \mathrm{~min}$; normal range: 5-10 min), coagulation FVIII had markedly decreased activity (12.6\%; normal range, 60-150\%), and FVIII inhibitor had a high titer [7.4 Bethesda units (BU)/ml; normal range, $0-0.6 \mathrm{BU} / \mathrm{ml}$ ]. Notably, the patient's autoantibody level was markedly higher
\end{abstract}

Correspondence to: Dr Zhuoyi Zhang, Department of Emergency, The First Affiliated Hospital of Zhejiang Chinese Medicine University, 54 Youdian Road, Hangzhou, Zhejiang 310006,P.R. China E-mail: zhangzhuoyi@zcmu.edu.cn

Abbreviations: FVIII, factor VIII; AHA, acquired hemophilia A; WBC, white blood cell; Hb, hemoglobin; CRP, C-reactive protein; PCT, procalcitonin; aPTT, activated partial thromboplastin time; FIB, fibrinogen; INR, international normalized ratio; $\mathrm{CD}$, cluster of differentiation; ANCA, antinuclear antibodies; IgM/IgA, immunoglobulin M/A; PPD, purified protein derivative; ANA, autoantibody; CT, computed tomography; TEG, thromboelastography; BU, Bethesda units; APCCs, activated prothrombin complex concentrates; LA, lupus anticoagulant

Key words: acquired hemophilia A, hemorrhage, premature delivery, polyserous effusions, China than normal $(1: 320$; normal range: $<1: 100)$. The patient was successfully treated with bleeding control, eradication of FVIII inhibitor, and treatment of the underlying disease. To the best of our knowledge, this is the first case of AHA with polyserous bloody effusions in a patient with an autoimmune disorder during the postpartum period. Reports of such rare cases will aid the characterization of disease pathogenesis, which may in turn lead to the recognition and timely treatment of this rare disorder.

\section{Introduction}

Acquired hemophilia A (AHA) is a clinically rare coagulopathy that results in soft tissue and mucocutaneous hemorrhage, and possible life-threatening bleeding (1). It has been reported that the annual incidence rate of AHA is $\sim 2$ individuals per million, worldwide (2). In the majority of cases, excessive bleeding episodes are spontaneous at presentation (2). The disorder presents without personal or family history of bleeding, and has a relatively high mortality rate, estimated at $9-22 \%$ (3).

To date, the pathogenesis of AHA has remained unclear. Only $\sim 50 \%$ of reported cases are typically associated with autoimmune disorders, malignancy, adverse drug reactions and/or various skin diseases (4). The optimal hemostatic therapy is controversial due to low incidence. The most common treatments for AHA with acquired factor VIII (FVIII) deficiency are bleeding management, eradication of the factor VIII inhibitor, treatment of underlying diseases, and decreasing the risk of injuries that may cause iatrogenic bleeding (2).

Herein, the present report documents a case of AHA with acquired FVIII deficiency associated with intra-abdominal hemorrhage, muscle hemorrhage and hemothorax 48 days after premature delivery. The diagnosis process included imaging and laboratory examinations. The patient was successfully diagnosed with AHA and treated with an infusion of prothrombin complex to prevent progressive bleeding and a glucocorticoid to eradicate the factor VIII inhibitor. Therefore, it may be proposed that it is important to differentiate AHA from another diseases, including congenital hemophilia A with inhibitors and lupus anticoagulants (LAs), 
which presents with spontaneous hemorrhaging or prolonged activated partial thromboplastin time (aPTT). Also presented is a review of AHA with regard to its epidemiology, associated risk factors, disease course and recent research concerning its management.

\section{Case report}

The current report was approved by the Ethics Committee of the First Affiliated Hospital of Zhejiang Chinese Medical University (Hangzhou, China). A 35-year-old woman who presented with persistent fever and right lower back pain for 4 days, as well as dizziness, right leg pain and right abdominal pain for 1 day, was admitted to the emergency department at the First Affiliated Hospital of Zhejiang Chinese Medical University on October 22, 2017. The patient had loss of sensation and diminished range of motion in the right leg, and transient loss of consciousness with hypotension. She had undergone childbirth 48 days prior to admission, and had no history of trauma or family history of hemopathy. The patient did have a history of appendicitis and no related surgeries.

On physical examination, abnormal vital signs included a blood pressure of $89 / 58 \mathrm{mmHg}$ (normal range, $90-140 / 60-90 \mathrm{mmHg}$ ) and temperature of $38^{\circ} \mathrm{C}$. In a lung examination, respiration was determined to be absent in the right lower lung. Shifting dullness was noted by percussion of the abdomen. The patient had severe pressure pain in the right lower abdomen, inner thigh and back, as well as percussion pain in the kidney region; furthermore, the right inner thigh appeared swollen. No peripheral edema or hepatosplenomegaly was noted.

A pelvic ultrasound confirmed a postpartum uterus and showed a suspicious teratoma in the left adnexal area, and a routine blood examination revealed a white blood cell (WBC) count of $10.1 \times 10^{9} / 1$ (normal range, 3.5-9.5 $\times 10^{9} / 1$ ), and hemoglobin (Hb; $118 \mathrm{~g} / \mathrm{l}$; normal range, 115-150 g/l) and platelet counts $\left(240 \times 10^{9} / 1\right.$; normal range, $\left.125-350 \times 10^{9} / 1\right)$ within the normal range, 3 days prior to admission (Hangzhou First People's Hospital; Huangzhou, China). The patient had no history of anticoagulation treatment or coagulopathy and no signs of inflammation of the abdomen, respiratory tract or urinary tract.

The patient underwent a series of examinations following admission, including for WBC count $\left(17.9 \times 10^{9} / 1\right)$, neutrophil granulocyte rate $(75.8 \%$; normal range, $40.0-75.0 \%), \mathrm{Hb}$ (70 g/l; normal range, 115-150 g/l), C-reactive protein (CRP; $73.09 \mathrm{mg} / \mathrm{l}$; normal range, 1-8mg/1), procalcitonin (PCT; $0.07 \mathrm{ng} / \mathrm{ml}$; normal range, 0-0.05 ng/ml), aPTT (68.4; normal range, 25.0-36.0 sec); D-dimer (1.37; normal range, $0-0.55 \mathrm{mg} / \mathrm{l})$ and fibrinogen [FIB; $4.11 \mathrm{~g} / \mathrm{l}$; normal range, $2.00-4.00 \mathrm{~g} / \mathrm{l}$; with a normal international normalized ratio (INR; 0.94; normal range, 0.8-1.2)]. Negative results were obtained for the serum tumor marker test, the Rous test which detected the metabolite of hemoglobin in urine to indicate that hemoglobinuria had been present recently, the Coombs test, complement regulatory protein cluster of differentiation (CD)55/CD59, human leucocyte antigen antibodies, the antinuclear antibodies (ANCA) profile, cytomegalovirus DNA, Epstein Barr virus DNA, virus immunoglobulin (Ig)M antibody, anticardiolipin $\operatorname{IgM}$ and $\operatorname{IgA}$, autoimmune hepatitis

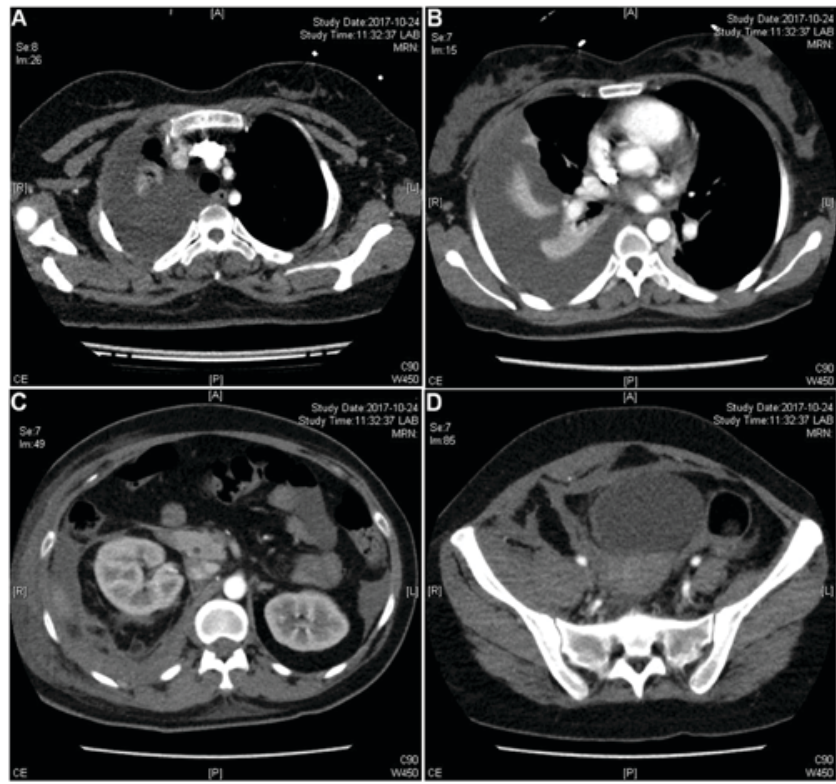

Figure 1. Contrast-enhancement computed tomography scan showing (A and B) pleural effusion, (C) right abdominal cavity effusion and (D) pelvic effusion and exudative lesions of the soft tissues surrounding the right groin and thigh.

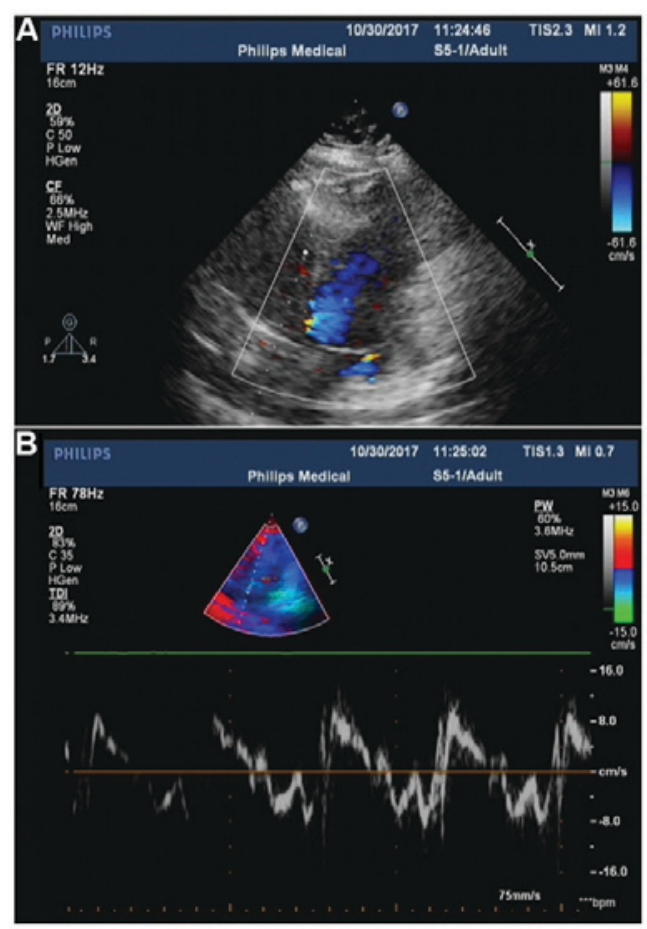

Figure 2. (A) Color doppler ultrasonography showing mild mitral valve reflux. (B) Tissue doppler imaging showing tricuspid valve regurgitation, high pulmonary artery systolic blood pressure and reduced diastolic left ventricular function. The parameters of cardiac function were as follows: Right ventricular internal dimension in diastole, $26.1 \mathrm{~mm}$; intraventricular septum in diastole, $7.6 \mathrm{~mm}$; left ventricular internal dimension-in diastole, $43.1 \mathrm{~mm}$; left ventricular posterior wall dimension, $7.6 \mathrm{~mm}$; left ventricular internal dimension in systole, $23.4 \mathrm{~mm}$; heart rate, $84 \mathrm{bpm}$; aorta, $23.1 \mathrm{~mm}$; left atrium, $32.5 \mathrm{~mm}$; fractional shortening, 45.7\%; ejection fraction, $77.4 \%$ ).

antibody, the tuberculin purified protein derivative (PPD) test, and rheumatoid factors. The patient's autoantibody (ANA) profile was 1:320 (normal range: $<1: 100$ ). 


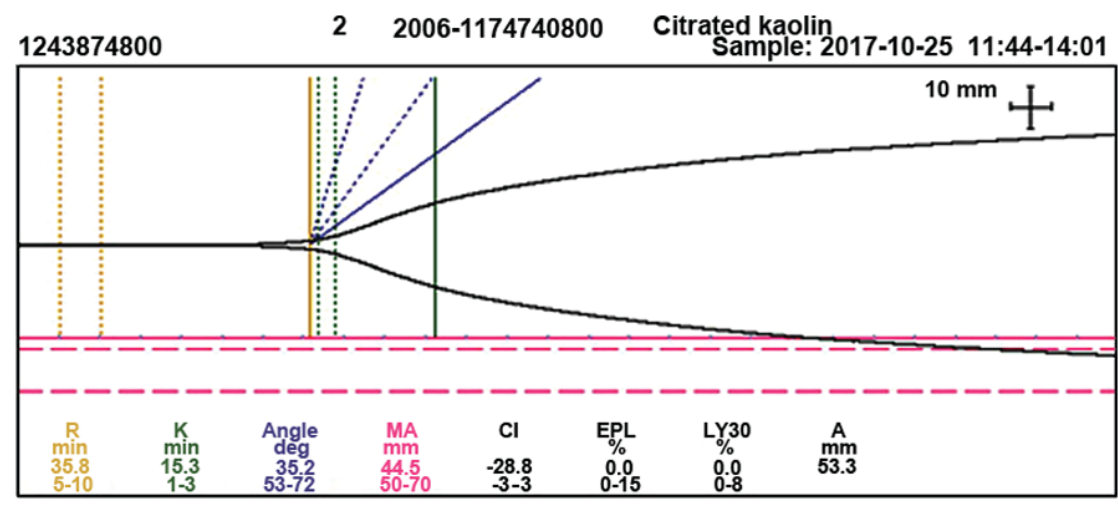

Figure 3. Thromboelastography showing a prolonged R-time $(35.8 \mathrm{~min})$ and K-time $(15.3 \mathrm{~min})$, a narrowed angle $\left(35.2^{\circ}\right)$, decreased MA (44.5 mm), decreased CI (-28.8) and normal clot lysis, which is reported in terms of EPL (0.0\%) and LY30\% (0.0\%). R, reaction time; K, coagulation time; deg, degree; MA, maximum amplitude; CI, coagulation index; EPL, estimated percent lysis; LY30\%, percent clot lysis at 30 min after maximum amplitude; A, amplitude.

Right pleural effusion, abdominal effusion and a dark liquid area in the posterior right abdomen and lumbar regions were observed during bedside ultrasonic testing. Abdominal and chest computed tomography (CT) revealed a markedly swollen right lumbar muscle, exudative lesions of the soft tissues surrounding the right groin, right thigh and right abdominal cavity, retroperitoneal and pelvic effusion, and right massive pleural effusion with incomplete expansion of the right lung (Fig. 1). Mild mitral valve reflux and tricuspid valve regurgitation, high pulmonary artery systolic blood pressure, and reduced diastolic left ventricular function were observed on heart doppler ultrasonography (Fig. 2).

The following were observed by thromboelastography (TEG): Reaction time (time from the start of the test to initial fibrin formation), $35.8 \mathrm{~min}$ (normal range, 5.0-10.0 $\mathrm{min}$ ), coagulation time (time required for clot formation), $15.3 \mathrm{~min}$ (normal range, 1.0-3.0 min), angle (measurement of clot growth), 35.2 (normal range, 53.0-72.0 ${ }^{\circ}$ ), maximum amplitude (measurement of clot strength), $44.5 \mathrm{~mm}$ (normal range, 50.0-70.0 $\mathrm{mm}$ ) and coagulation index, -28.8 (normal range, -3.0-3.0; Fig. 3). Markedly decreased FVIII activity (12.6\%; normal range, 60.0-150.0\%), decreased FVIII procoagulant activity (FVIII: C; 0.8\%; normal range, 50.0-150.0\%) and a high-titer of the FVIII inhibitor [7.4 Bethesda units $(\mathrm{BU}) / \mathrm{ml}$; normal range, 0-0.6 $\mathrm{BU} / \mathrm{ml}$ ] was observed in a coagulation factor assay.

Collectively these examinations indicated that the patient had AHA. Antishock and coagulation factor replacement were provided to stop the bleeding. A transfusion of red blood cells, plasma, prothrombin complex (PCC) and vitamin K was initiated during the first 4 days of hospitalization. Due to concerns about the patient's high WBC count and CRP and PCT levels, the antibiotic meropenem was administered at $1.0 \mathrm{~g}$ every $12 \mathrm{~h}$, to prevent potential infections. Following a suspected diagnosis of AHA, the patient was treated with intravenous human coagulation FVIII (1,600 IU twice daily), dexamethasone $(5 \mathrm{mg} /$ day $)$ and intravenous Ig (10 g/day). Thoracic drainage for 7 days relieved the chest congestion, which was determined to be exudative bloody pleural effusion, based on cell counting and classification of the pleural effusion under the microscope, as well as the Rivalta test (color, red; Rivalta test, positive; WBC, $600.0 \mu 1$; neutrophil percentage, $46.0 \%$; lymphocytes percentage, $12.0 \%$; mesothelial cells percentage, $42.0 \%$ ). One week later, the patient experienced pain relief and normal body temperature was recovered; in addition, the $\mathrm{Hb}$ levels had risen $(85 \mathrm{~g} / \mathrm{l}$; normal range, $115-150 \mathrm{~g} / \mathrm{l}$ ) and the extensive subcutaneous ecchymoses of the right thigh and lower back had disappeared. Therefore, the glucocorticoid treatment was changed to oral prednisone, $20 \mathrm{mg}$ three times a day, and then to oral prednisone, $15 \mathrm{mg}$ three times a day, 2 weeks later. Concurrently, the patient was also administered calcitriol soft capsules (R.P. Scherer $\mathrm{GmbH}$ \& Co. KG, Eberbach, Germany), 1 capsule $(0.25 \mu \mathrm{g})$ twice a day, and calcium carbonate and vitamin D3 tablets (Wyeth pharmaceuticals, Inc., Collegeville, PA, USA), 1 pill (calcium carbonate $1.5 \mathrm{~g}$ and vitamin D3 $125 \mathrm{IU}$ ) twice a day, to prevent osteoporosis due to the glucocorticoid. At 1 month after treatment, the pain and subcutaneous ecchymosis in the patient's right side were relieved, a chest CT scan showed clearing of the exudative lesions, and laboratory values gradually recovered, including those of a routine blood examination, aPTT, FIB and D-dimer levels, and the level of FVIII inhibitor was reduced (0 BU/ml).

The patient was discharged on November 17, 2017. Her discharge medication consisted of prednisone, $10 \mathrm{mg}$ three time a day, calcitriol soft capsules, 1 capsule twice a day, and calcium carbonate and vitamin D3 tablets, 1 pill twice a day. Moreover, we suggested weekly follow-ups. There was no indication of relapse at the 2-month follow-up.

\section{Discussion}

In the present case, a rapid accurate diagnosis was a prerequisite for successful treatment. Patients with polyserous effusions typically visit an oncologist, surgeon or respiratory physician (5-7). In the absence of trauma history and a family history of bleeding disorders in the present case, as well as negative results on tumor protein expression analysis and tuberculin PPD testing, genetic diseases, malignancy and tuberculosis were excluded. Therefore, hematopoietic diseases were considered. The patient's aPTT was elevated with normal INR and normal prothrombin time. We suspected endogenous coagulation dysfunction or LA. The lack of aPTT correction following a mixing test was cause for suspected AH or LA.

The TEG analysis indicated a coagulation factor abnormality. Following consideration of the above results along 


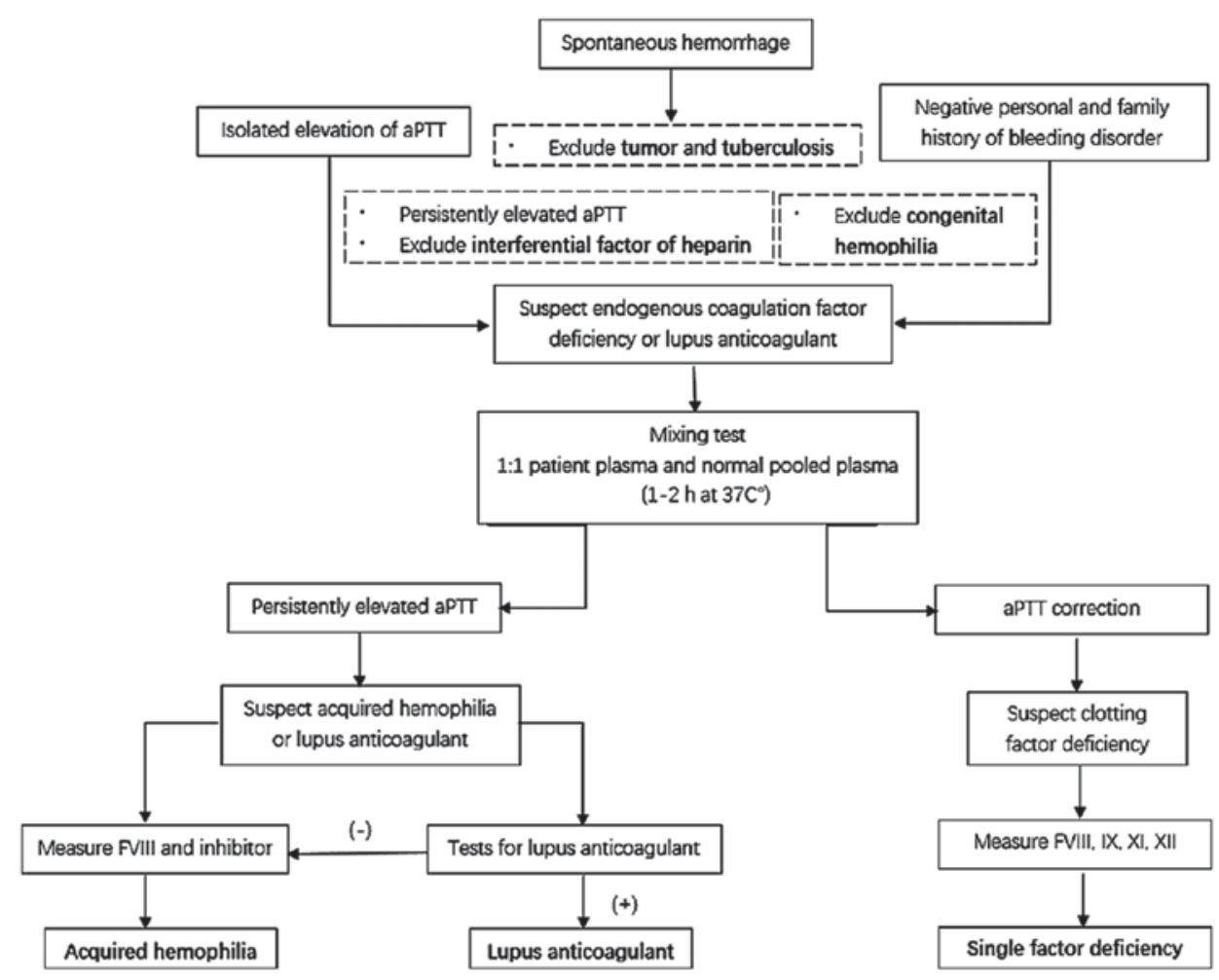

Figure 4. Schematic flowchart for the diagnosis of acquired hemophilia, lupus anticoagulant and single factor deficiency. FVIII, factor VIII; aPTT, activated partial thromboplastin time.

with the markedly decreased FVIII activity, it was inferred that the patient's pathology was associated with FVIII deficiency. Moreover, the presence of FVIII inhibitor verified the suspected AHA (Fig. 4).

It is necessary to differentiate AHA from congenital hemophilia A with inhibitors, since the latter indicates a personal and family history of bleeding disorders since childhood and exhibits clinical characteristics that include muscle and joint spontaneous hemorrhage and joint deformity; moreover, it is consistent with the $\mathrm{X}$-linked recessive inheritance law (8). The same antibody produced by a hemophilia A patient may completely inactivate FVIII without residual FVIII: C (2). Therefore, the hemostatic effect is not produced following infusion of FVIII preparations at the same dose that may have been effective previously (9).

In addition, LA presents with a persistent prolonged aPTT, as does AHA, and may lead to the false appearance of reduced coagulation factors in vitro due to its inhibitory effect on phospholipids (10). In LA, prolonged aPTT cannot be corrected by using normal plasma; however, it can be shortened and corrected by the supplementation of exogenous phospholipids (11). This can be proven more definitively through a variety of phospholipid-dependency experiments and by performing the Dilute Russell's viper venom time test. FVIII autoantibodies and LA may coexist in the same patient $(11,12)$. For complicated cases, ELISA may be used to identify FVIII inhibitors in LA (12). An aPTT reagent, which is not sensitive to LA, can be used to eliminate its effect on coagulation (2). Clinically, patients with LA primarily manifest with thrombosis events, and bleeding is rarely observed $(12,13)$.
Although there is reportedly no significant difference in the incidence of AHA between the sexes, it is predominantly a disease of the elderly aged $\geq 60$ years (7). However, it can also be associated with pregnancy and autoimmune disease in younger groups (aged 20-30 years) $(2,14)$. Most older patients with AHA tend to be women, while most younger patients tend to be men $(14,15)$.

Reports on postpartum AHA are rare. In a previous study, $\sim 10 \%$ of AHA cases were associated with pregnancy (16). The hemorrhagic symptoms in female patients are commonly present between 1 and 4 months after parturition $(17,18)$.

AHA is often associated with autoimmune disease, including rheumatoid arthritis, systemic lupus erythematosus and myasthenia gravis (2). The incidence of AHA associated with autoimmune disorders has been demonstrated to be as high as $20 \%$ in all AHA patients, worldwide (19). High FVIII inhibitor titers often appear in patients with autoimmune disorders as well as pregnant individuals (2). However, in postpartum subjects, the hemorrhagic potential is often low and the inhibitors spontaneously disappear in almost all of these patients, with titers of inhibitors lower than those of patients with an autoimmune disorder (17). Furthermore, it may be difficult to achieve inhibitor eradication in patients with high titers ( $\geq 5 \mathrm{BU} / \mathrm{ml}$ ), even with aggressive immunosuppressive therapy (2). The pathogenesis may be that carrying a fetus could induce the risk of fatal bleeding since it induces the risk of diaplacental transition and the development of postpartum inhibitors in pregnant AHA patients (2). Therefore, the presence of an autoimmune disease should be considered in this patient and with 
continued monitoring of ANA and ANCA profiles to detect autoimmune pathogens.

AHA is an important disorder clinically and economically, and often unrecognized or misdiagnosed, which may be reason for its relatively high mortality rate, estimated to be $\sim 30 \%$, worldwide $(16,20)$. Therefore, aggressive treatment is recommended to decrease the mortality rate in AHA (14). The main goals of AHA management are to control and prevent bleeding, eradicate the FVIII inhibitor and treat the underlying disease. The first-line treatment for severe bleeding episodes is administration of bypassing agents, including activated prothrombin complex concentrates (APCCs) containing factors II, VII, IX, X and VIIa, human FVIII and recombinant activated factor VII (5). In the present case, FVII was not administered because of its high cost and the beneficial therapeutic effect that was provided by administration of APCC and FVIII. Agents used in immunosuppressive therapy for suppressing the FVIII inhibitor include long-term immunosuppressive agents, consisting of corticosteroids alone or in combination with azathioprine, cyclosporine and antineoplastic agents, including cyclophosphamide, mercaptopurine and vincristine $(2,6)$. The use of intravenous immunoglobulin in multiple treatment sessions is another eradication strategy; however, its effects remain unclear.

Infectious diseases, including pneumonia and sepsis, are responsible for $\sim 50 \%$ of AHA-associated mortalities (21). Therefore, sufficient attention to the prevention and early detection of infectious diseases is warranted when aggressive and prolonged immune suppression therapy is provided (2). For these reasons, antimicrobial agents were also prescribed presently.

In conclusion, the current report describes, to our knowledge, the first reported case of AHA with a possible autoimmune disorder during the postpartum period in a 35-year-old female who presented with polyserous bloody effusions. Awareness of the correct diagnosis of AHA is necessary as it is a curable disease, although it can be life threatening. It was also highlighted how timely treatment can be successful and lifesaving. Corticosteroids remain the most commonly prescribed immunosuppressive therapy treatment for AHA patients, in addition to bleeding management and treatment of the underlying disease.

\section{Acknowledgements}

The authors would like to thank Editage for the scientific editing of the manuscript, and the staff at the Departments of Hematology and Emergency, The First Affiliated Hospital of Zhejiang Chinese Medical University for their help in the management of this patient.

\section{Funding}

The present study was supported by grants from the National Natural Science Foundation of China (grant no. 81503522) and Zhejiang Natural Science Foundation (grant no. LQ15H270004).

\section{Availability of data and materials}

All data generated or analyzed during this study are included in this published article.

\section{Authors' contributions}

LX and ZZ made substantial contributions to conception and design and revised the manuscript critically. XZ, JC and $\mathrm{XH}$ made the contributions to acquisiton of data and wrote the draft. YT, NZ and LW interpreted the results and were involved in drafting the manuscript and revising it critically. All authors have read and given final approval of version to be published and agreed to be accountable for all aspects of the work in ensuring that questions related to the accuracy or integrity of any part of the work are appropriately investigated and resolved.

\section{Ethics approval and consent to participate}

This case was approved by the Ethics Committee of the First Affiliated Hospital of Zhejiang Chinese Medical University.

\section{Patient consent for publication}

Authors have disclosed to this patient that personally identifiable material would be available via the Internet as well as in print after publication. Written informed consent was obtained from the patient under these conditions.

\section{Competing interests}

The authors declare that they have no competing interests.

\section{References}

1. Vautier M, de Boysson H, Creveuil C, Repesse Y, Borel-Derlon A, Troussard X, Damaj GL, Bienvenu B, Gautier P and Aouba A: Influence of factor VIII level and its inhibitor titer on the therapeutic response to corticosteroids alone in the management of acquired hemophilia. Medicine (Baltimore) 95: e5232, 2016.

2. Sakurai Y and Takeda T: Acquired hemophilia A: A frequently overlooked autoimmune hemorrhagic disorder. J Immunol Res 2014: 320674, 2014

3. Collins P, Baudo F, Huth-Kühne A, Ingerslev J, Kessler CM, Castellano MEM, Shima M, St-Louis J and Lévesque H: Research article Consensus recommendations for the diagnosis and treatment of acquired hemophilia A: BMC Research Notes 3: 161, 2010.

4. Aljasser MI, Sladden C, Crawford RI and Au S: Bullous pemphigoid associated with acquired hemophilia A: A rare association of autoimmune disease. J Cutan Med Surg 18: 123-126, 2014.

5. Olteanu M, Niţu M and Golli A: Tuberculosis mesenteric adenopathy and polyserositis. Rom J Morphol Embryol 53: 835-840, 2012.

6. Kruse-Jarres R, Kempton CL, Baudo F, Collins PW, Knoebl P, Leissinger CA, Tiede A and Kessler CM: Acquired hemophilia A: Updated review of evidence and treatment guidance. Am J Hematol 92: 695-705, 2017.

7. Bitting RL, Bent S, Li Y and Kohlwes J: The prognosis and treatment of acquired hemophilia: A systematic review and meta-analysis. Blood Coagul Fibrinolysis 20: 517-523, 2009.

8. Chinese society of hematology: Chinese expert consensus on the diagnosis and treatment of acquired hemophilia A. Zhonghua Xue Ye Xue Za Zhi 35: 575-576, 2014 (In Chinese).

9. Coppola A, Franchini M, Castaman G, Santagostino E, Santoro C, Santoro RC, Morfini M, Minno GD, Rocino A and on behalf of the AICE ad hoc Working Group: Treatment regimens with bypassing agents in patients with hemophilia A and inhibitors: A survey from the Italian association of hemophilia centers (AICE). Semin Thromb Hemost 44: 551-560, 2018.

10. Brandt JT, Barna LK and Triplett DA: Laboratory identification of lupus anticoagulants: Results of the second international workshop for identification of lupus anticoagulants. Thromb Haemost 74: 1597-1603, 1995. 
11. Kumano O, Amiral J, Dunois C, Peyrafitte M and Moore GW: Paired APTTs of low and high lupus anticoagulant sensitivity permit distinction from other abnormalities and achieve good lupus anticoagulant detection rates in conjunction with dRVVT. Int J Lab Hematol 24, 2018.

12. Thrombosis and Hemostasis Group, Chinese Society of Hematology, Chinese Medical Association: Chinese expert consensus on the diagnosis and treatment of acquired hemophilia A (Chinese). Chin J Hematol 35: 575-576, 2014.

13. Greaves M, Cohen H, MacHin SJ and Mackie I: Guidelines on the investigation and management of the antiphospholipid syndrome. BrJ Haematol 109: 704-715, 2000

14. Theodossiades G, Tsevrenis V, Nomikou E, Dadiotis L and Kontopoulou-Griva L: Surgery-associated acquired hemophilia A. Ann Hematol 80: 691-693, 2011.

15. Bouvry Pand Recloux P: Acquired hemophilia.Haematologica 79: 550-556, 1994

16. Sborov DW and Rodgers GM: Acquired hemophilia A: A current review of autoantibody disease. Clinical Advances in Hematol Oncol 10: 19-27, 2012.

17. Delgado J, Jimenez-Yuste V, Hernandez-Navarro F and Villar A: Acquired haemophilia: Review and meta-analysis focused on therapy and prognostic factors. Br J Haematol 121: 21-35, 2003.
18. Collins PW and Percy CL: Advances in the understanding of acquired haemophilia A: Implications for clinical practice. $\mathrm{Br}$ J Haematol 148: 183-194, 2010.

19. Shetty S, Bhave M and Ghosh K: Acquired hemophilia A: Diagnosis, aetiology, clinical spectrum and treatment options. Autoimmun Rev 10: 311-316, 2011.

20. Federici AB, Budde U, Castaman G, Rand JH and Tiede A: Current diagnostic and therapeutic approaches to patients with acquired von Willebrand syndrome: A 2013 update. Semin Thromb Hemost 39: 191-201, 2013.

21. Tanaka I, Amano K, Taki M, Oka T, Sakai M, Shirahata A, Takata N, Takamatsu J, Taketani H, Hanabusa H, et al: A 3-year consecutive survey on current status of acquired inhibitors against coagulation factors in Japan: Analysis of prognostic factors. Japanese J Thromb Hemost 19: 140-153, 2008.

(i) $(-)$ This work is licensed under a Creative Common Attribution-NonCommercial-NoDerivatives 4.0 International (CC BY-NC-ND 4.0) License. 